\title{
Natural Coinfection of Pseudomonas aeruginosa and Enteroaggregative Escherichia coli in a feral pigeon (Columba livia)
}

\author{
Ruben Horn Vasconcelos', Windleyanne Gonçalves Amorim Bezerra', Raul Antunes Silva Siqueira', \\ Pedro Henrique Quintela Soares de Medeiros' ${ }^{2}$, Ricardo Barbosa Lucena ${ }^{3}$, Alexandre Havt ${ }^{2}$, \\ Isaac Neto Goes da Silva' \& William Cardoso Maciel'
}

\begin{abstract}
Background: Rock pigeon (Columba livia) is an exotic and invasive species of the Columbidae family found in several cities around the world, including Brazilian cities, and close to humans. This species is found in large populations often in public locations, where people may have contact. Several studies have demonstrated the isolation of human pathogens from these birds. However, there are scarce studies describing infections by Gram-negative bacteria to which pigeons are susceptible. Therefore, this report aimed to describe a case of natural coinfection of Pseudomonas aeruginosa and Enteroaggregative Escherichia coli in a feral pigeon.

Case: A sick feral pigeon was delivered at the Laboratory of Ornithological Studies, State University of Ceará, Fortaleza, Brazil. Due to poor prognosis, the individual was euthanized with ketamine via intravenous injection and submitted to necropsy, in which samples were collected for microbiological and histopathological procedure. The procedure was performed aseptically and samples were collected from intestine, liver, spleen, heart and lung. Bacterial isolation was performed with culture media selective for Gram negative bacteria and strains were identified biochemically. Histopathological examination was performed with conventional method and slides were stained with hematoxylin and eosin. DNA from $E$. coli isolates was extracted with simples boiling method and submitted to uniplex conventional polymerase chain reaction (PCR) to diagnose diarrheagenic pathotypes with specific primers for the following genes: aaiC, aatA, eaeA, stxl, stx2, eltB, estA and ipaH. Escherichia coli was isolated from the intestine, liver, spleen and lung, while Pseudomonas aeruginosa was present in liver, spleen and lung. E. coli strains from liver and spleen were positive for the aaiC gene, which is a diagnostic gene for Enteroaggregative Escherichia coli (EAEC) pathotype. Necropsy revealed the presence of several caseous lesions around the head of the bird, cellulitis in the abdominal region, hepatomegaly, splenomegaly, hemorrhagic intestine and pericarditis. Microscopical findings were most intense in liver and lung samples, presenting vascular and inflammatory alterations. Histopathological alterations indicated an acute presentation of the infections and several lesions in different organs, demonstrating the septicemic characteristic of the microorganisms involved in this case.

Discussion: Both pathogens in this report were identified mostly in the same organs, which may indicate that there was an association in the pathogenesis. However, the entry routes of infection in this case may have been different, considering that Pseudomonas aeruginosa was not isolated from the intestine. The histopathological findings were not pathognomonic for either pathogen. However, some characteristic lesions were observed, such as cellulitis, which is commonly attributed to Escherichia coli. EAEC strains are human pathogens that cause acute and persistent diarrhea around the world. These microorganisms could be potentially transmitted to humans, as suggested by other studies with feral pigeons and enteric pathogens. However, the absence of reports of pigeon-transmitted diarrheagenic infections in humans may suggest that pigeons are only reservoirs of these pathogens. In addition, as this report demonstrates, these birds may also suffer from these infections. In conclusion, the free-living pigeon presented a natural occurring case of coinfection by Pseudomonas aeruginosa and Enteroaggregative Escherichia coli. Both are human opportunistic pathogens, and may have public health implications.
\end{abstract}

Keywords: rock pigeon, extra-intestinal infection, wild birds, EAEC. 


\section{INTRODUCTION}

Feral pigeons are classified in the species Columba livia and are one of the main species of the Columbidae family, Columbiformes order. The populations of this species currently found in most cities are derived from individuals captured and domesticated at least five thousand years ago in their natural region [5]. However, people started to care for these birds and feed them, which along with the available food found inside the cities and the lack of natural predators provided a propitious environment for these birds to adapt. Currently, feral pigeons are found in numerous populations in public locations, often in close contact with man $[2,3]$.

At least 60 different human pathogens have been already identified in these birds. However, studies performed with feral pigeons aiming to assess their zoonotic potential to transmit diseases to humans are limited to the isolation of microorganisms [4], not considering pathological aspects of the infection in these birds. Despite being frequently isolated, the reports of transmission of such pathogens to humans from pigeons are scarce, usually occurring with immunosuppressed individuals. Currently, there are no reports of coinfection with Pseudomonas aeruginosa and Enteroaggregative Escherichia coli (EAEC) in pigeons, or even adequate reports of isolated infections by each of these pathogens in this avian species. Therefore, this study aimed to describe a case of natural occurring coinfection of Pseudomonas aeruginosa and Escherichia coli in a freeliving pigeon (Columba livia) from Fortaleza, Brazil.

\section{CASE}

A free-living pigeon was found sick and delivered to the Laboratory of Ornithological Studies, State University of Ceará (UECE), Fortaleza - Brazil. Clinical evaluation revealed that the bird presented poor body condition, intense diarrhea, lesions around the head, mostly close to eyes and ears. Due to the advance state of disease, poor prognosis, and the possibility of a zoonotic infection, euthanasia was performed three days after admission with an intravenous injection of ketamine $(1 \mathrm{~mL})$. Necropsy was performed for macroscopic observation, sampling collection for microbiological procedure for bacterial isolation and histopathological analysis of organs. Procedure was performed close to the Bunsen burner with sterile equipment and samples were aseptically collected from intestine, liver, spleen, heart and lung. For histopathological examination, frag- ments of organs were placed in tubes with 5\% formalin, submitted for standard histological procedure using paraffin and thin cuts stained with hematoxylin-eosin observed in simple light microscope.

For bacterial isolation, samples were placed in tubes containing BHI broth, submitted for incubation and aliquots were collected and plated in EosinMethylene-Blue (EMB) agar. Colonies were selected based on morphological characteristics and submitted for biochemical identification. Isolates were sent to the Laboratory of Enterobacteria (LABENT/FIOCRUZ), which is a reference for confirming the identification. E. coli strains were submitted to conventional uniplex Polymerase Chain Reaction (PCR) technique for identification of 5 out of 6 diarrheagenic pathotypes currently described.

DNA from the isolates was extracted by simple method of boiling in Triton X-100, centrifugation and quantification of the supernatant in spectrophotometer. For the uniplex PCR, GoTaq Green Master Mix kit was used and specific primers previously described were used for the diagnosis of diarrheagenic pathotypes: genes stx 1 (348pb) and stx2 (584pb) for the identification of ShigaToxin producing E. coli (STEC); eltB (508pb) and estA (147pb) for Enterotoxigenic E. coli (ETEC); eaeA (881 $\mathrm{pb})$ for Enteropathogenic E. coli (EPEC); ipaH (483 pb) for enteroinvasive E. coli (EIEC); aatA (630 pb) and aaiC (215pb) for Enteroaggregative E. coli (EAEC) [10]. Strains EAEC 042, EHEC O157:H7, EIEC O124, EPEC 2348/69 and ETEC H10407 were used as positive controls for the reactions. Amplified products were submitted to $2 \%$ agarose gel electrophoresis stained with ethidium bromide and revealed in transilluminator.

Macroscopic findings are displayed in Figure 1. Necropsy confirmed the poor body condition, revealing cachexia. In addition, the presence of several caseous masses around the head of the bird, cellulitis in the abdominal region, diarrheal feces around cloacae, hepatomegaly, splenomegaly, hemorrhagic intestine and fibrinous pericarditis were observed.

Microscopic findings are displayed in Figure 2. Histopathological analysis revealed a diffuse congestion in lung slides with some vessels obliterated by fibrin thrombi. In addition, multiple granulomas were observed in parenchyma, characterized by necrotic cell agglomerates circled by multinucleated giant cells (Figure 2A). Liver samples presented a diffuse and intense congestion of sinusoids in addition to multiple random foci of histiocytic inflammation associated 
with hepatocyte necrosis (Figure 2B). Many of these inflammation foci coalesced and, in some, the presence of heterophils and lymphocytes was observed. Deep dermis and subcutaneous revealed a mixed infiltrate of heterophils, lymphocytes, plasma cells and histiocytes, associated with a great amount of fibrin, edema and numerous basophilic bacterial aggregates with granular aspect (cellulitis). Vessels from leptomeninges of cerebrum and cerebellum were congested. Lamina propria from the intestines presented moderate and diffuse infiltration by lymphocytes and plasma cells.

Bacterial isolation demonstrated the presence of Escherichia coli in intestine, liver, spleen and lung, and Pseudomonas aeruginosa in liver, spleen and lung. The E. coli isolates from liver and spleen presented an amplicon of approximately $215 \mathrm{bp}$ compatible with aaiC gene and were negative for the remaining genes.

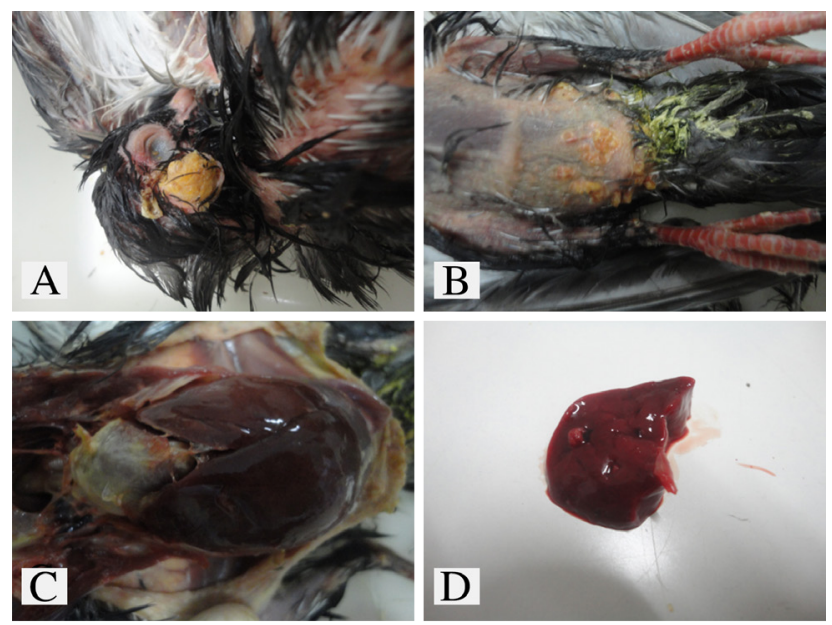

Figure 1. Macroscopic findings in necropsy of a feral pigeon (Columba livia) with coinfection of Pseudomonas aeruginosa and Enteroaggregative Escherichia coli. Caseous masses (A) and cellulitis (B) were observed in skin, in addition to diarrheal feces around cloacae. Hepatomegaly and fibrinous pericarditis (C) were observed in coelomic cavity and after cutting the liver, a granuloma was identified (D).

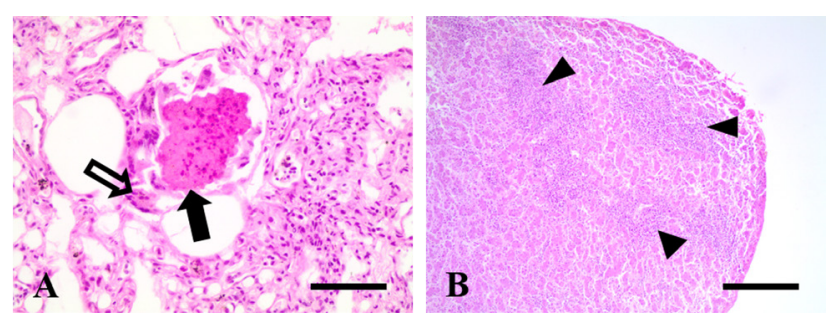

Figure 2. Histopathological findings in liver and lung samples of feral pigeon with t of Pseudomonas aeruginosa and Enteroaggregative Escherichia coli. A- Lung, granuloma with necrotic cells (arrow) surrounded by giant multinucleated cells (outlined arrow). [Bar= 50 $\mu \mathrm{m}]$. B- Liver, random foci of histiocytic inflammation (arrowheads). [Bar $=100 \mu \mathrm{m}]$.

\section{DISCUSSION}

In this report, a feral pigeon was submitted to necropsy, in which a coinfection of Pseudomonas aeruginosa and Escherichia coli was identified. Both pathogens were isolated from liver, spleen and lung, with the exception of the intestine, in which only E. coli was found. The lesions observed in the histopathological analysis are not pathognomonic and the causative agent for each one may not be confirmed. However, Escherichia coli is one of the main pathogens that cause cellulitis in poultry [7], while Pseudomonas aeruginosa is not associated with this type of lesion, which suggests that this finding was caused by $E$. coli. The several lesions observed in extra-intestinal organs with the presence of granulomas, along with the isolation results, demonstrate that there was septicemia by both microorganisms, which may penetrate by different entry routes. The absence of Pseudomonas aeruginosa in the intestine suggest that this bacterium penetrated via another path. However, this hypothesis could not be confirmed with the methodology applied in this report.

Two out of the four E. coli isolates were positive for the aaiC gene, which is important in the VI type secretion system in Enteroaggregative Escherichia coli (EAEC) [1]. In addition, this gene is used in the diagnosis for this pathotype in human samples [6]. This finding reveals a risk for the public health, considering this pathotype is diarrheagenic and frequently cause acute and persistent diarrhea in several locations around the world [8]. Interestingly, when compared to other intestinal pathogens, EAEC presents one of the biggest prevalence rates in human samples and is often associated with asymptomatic infections [9]. However, identifying this pathogen in extra-intestinal samples of avian origin is unprecedented, considering EAEC is most frequently found in intestinal or fecal samples of human origin.

The results presented in this report suggest that both microorganisms invaded the organism in association causing septicemia, since both were found mostly in the same organs. The interaction between microorganisms of different species occurs mainly by quorum sensing mechanisms and several of these have been described in E. coli [12] and in Pseudomonas aeruginosa [11]. Therefore, it is possible that this type of interaction have promoted the coinfection in this case, activating the expression of virulence genes for the development of 
the disease, since both of the microbial species involved in this case are known opportunistic pathogens.

In conclusion, this report presented an extra-intestinal case of coinfection by Pseudomonas aeruginosa and Enteroaggregative Escherichia coli (EAEC) in a feral pigeon. These results suggest that pigeons (Columba livia) are capable of hosting these pathogens and are susceptible to their infection, especially when associated.
Funding. During this study, the first author had a doctoral scholarship provided by the Fundação Cearense de Apoio ao Desenvolvimento Científico e Tecnológico (FUNCAP).

Acknowledgments. We thank the Laboratory of Ornithological Studies (LABEO) for the technical support.

Declaration of interest. The authors report no conflicts of interest. The authors alone are responsible for the content and writing of the paper.

\section{REFERENCES}

1 Dudley E.G., Thomson N.R., Parkhill J., Morin N.P. \& Nataro J.P. 2006. Proteomic and microarray characterization of the AggR regulon identifies a phe U pathogenicity island in enteroaggregative Escherichia coli. Molecular Microbiology. 61: 1267-1282.

2 Haag-Wackernagel D. 1993. Street pigeons in Basel. Nature. 361: 200.

3 Haag-Wackernagel D. 1995. Regulation of the street pigeon in Basel. Wildlife Society Bulletin. 23: 256-260.

4 Haag-Wackernagel D. \& Moch H. 2004. Health hazards posed by feral pigeons. Journal of Infection. 48: 307-313.

5 Johnston R. F. 1998 Feral pigeons. The Kansas School Naturalist. 42(2): 1-15.

6 Lima I.F.N., Quetz J.S., Guerrant R.L., Nataro J.P., Houpt E.R., Lima A.A.M. \& Havt A. 2013. Enteroaggregative Escherichia coli quantification in children stool samples using quantitative PCR. APMIS. 121: 643-651.

7 Ngeleka M., Kwaga J.K.P., White D.G., Whittam T. S., Riddell C., Goodhope R., Potter A.A. \& Allan B. 1996. Escherichia coli Cellulitis in Broiler Chickens: Clonal Relationships among Strains and Analysis of Virulence-Associated Factors of Isolates from Diseased Birds. Infection and Immunity. 64(8): 3118-3126.

8 Okhuysen P.C. \& Dupont H.L. 2010. Enteroaggregative Escherichia coli (EAEC): a cause of acute and persistent diarrhea of worldwide importance. Journal of Infectious Disease. 4: 503-505.

9 Platts-Mills J.A, Babji S., Bodhidatta L., Gratz J., Haque R., Havt A., McCormick B.J., McGrath M., Olortegui M.P., Samie A., Shakoor S., Mondal D., Lima I.F.N., Hariraju D., Rayamajhi B.B., Qureshi S., Kabir F., Yori P.P., Mufamadi B., Amour C., Carreon J.D., Richard S.A., Lang D., Bessong P., Mduma E., Ahmed T., Lima A.A.A.M., Mason C.J., Zaidi A.K.M., Bhutta Z.A., Kosek M., Guerrant R.L., Gottlieb M., Miller M., Kang G. \& Houpt E.R. 2015. Pathogen-specific burdens of community diarrhoea in developing countries: a multisite birth cohort study (MAL-ED). Lancet Global Health. 3: 564-575.

10 Taniuchi M., Walters C.C., Gratz J., Maro A., Kumburu H., Serichantalergs O., Sethabutr O., Bodhidatta L., Kibiki G., Toney D.M., Berkeley L., Nataro J.P. \& Houpt E.R. 2012. Development of a multiplex polymerase chain reaction assay for diarrheagenic Escherichia coli and Shigella spp. and its evaluation on colonies, culture broths, and stool. Diagnostic Microbiology and Infectious Disease. 73: 121-128.

11 Venturi V. 2006. Regulation of quorum sensing in Pseudomonas. FEMS Microbiology Reviews. 30: 274-291.

12 Walters M. \& Sperandio V. 2006. Quorum sensing in Escherichia coli and Salmonella. International Journal of Medical Microbiology. 296: 125-131. 